\title{
Sleep Induced by a Herbal Agent Polygala tenuifolia versus that by Alprazolam: A Review with New Data on EEG and Sleep Duration
}

\author{
Juan Yu', Tony CH Chow ${ }^{2,4}$, Sharon L Wu ${ }^{2,4}$, HM Cheung ${ }^{2,4}$, Fei Hong ${ }^{1, *}$, David T Yew ${ }^{2,3,4,{ }^{*},+}$
}

\begin{abstract}
This study is the first comparison of an herbal agent with a well-established hypnotic for sleep. The beneficial effects for sleep of the herb Polygala tenuifolia (with main sedative components being 3,4-methoxycinnamic acid and phenylpropanoids) and Alprazolam were investigated, and our results indicated that both could benefit sleep via the shortening of sleep induction time and the increase in wave forms of electroencephalogram (EEG) associated with stages 1, 2 and 3 of the non-rapid eye movement (NREM) sleep. Increase of numbers of theta waves, $\mathrm{v}$ waves, delta waves and $\mathrm{k}$ complex were observed in mice, indicating deeper and better quality of sleep. There was an obvious increase in the duration of theta plus delta waves in both Polygala and Alprazolam treated groups, while the wave duration was slightly longer in the Polygala group than the Alprazolam group. When the Alprazolam treated group was added with Polygala, the theta plus delta wave duration increased and exceeded that from Alprazolam treatment alone. Similarly, increase of duration of $\mathrm{k}$ complex was also observed in both Polygala and Alprazolam treated groups. This study illustrated that the herbal agent Polygala tenuifolia could have at least similar efficacy in the induction of sleep as the manufactured drug Alprazolam.
\end{abstract}

\section{Keywords}

Sleep, Herbal medicine, Polygala tenuifolia, Alprazolam, Electroencephalogram

\begin{abstract}
Introduction
There have been studies of actions on herbal medicine on the nervous system and Polygala tenuifolia is one of the phytochemicals that is known for its effects on the nervous system [1, 2,3]. The genus Polygala is an angiospermic plant located in particular in Asia, from the mid part of China to Mongolia and Russia. It is also cultivated and used in India and Korea and cultivated in Canada and Brazil in smaller quantities [4]. In India, Polygala had not only
\end{abstract}

been an allopathic medicine for hypoglycemia, but also an anticancer agent, a CNS tranquillizer and a hypolidemic agent [4]. The root of a species of Polygala tenuifolia is used specially as an herbal medicine in China under the name Yuan Zhi for thousands of years. The main components of this herb were clearly deciphered chemically and the characteristic ones include tenuifolin, polygalaxanthone III, and 3, 6'-disinapoly sucrose [5], while $\mathrm{Xu}$ et al. identified in detail the xanthones: 7-hydroxy-1 -methoxy-2,3-

'Fujian Provincial Key Laboratory of Pien Tze Huang Natural Medicine Research and Development (R \& D), Zhangzhou Pien Tze Huang Pharmaceutical CO., LTD. Zhangzhou, China

${ }^{2} \mathrm{School}$ of Chinese Medicine, The Chinese University of Hong Kong, Shatin, N.T., Hong Kong

${ }^{3}$ School of Biomedical Sciences, The Chinese University of Hong Kong, Shatin, N.T., Hong Kong

${ }^{4}$ Hong Kong College of Technology, 2 On Shing Street, Ma On Shan, Shatin, Hong Kong

${ }^{\dagger}$ Author for correspondence: David T Yew, School of Biomedical Sciences, The Chinese University of Hong Kong, Shatin, N.T., Hong Kong, Tel: 852 39434140; Fax: 852 39420941; email: david-yew@cuhk.edu.hk 
methylenedioxyxanthone(1), 1, 7-dihydroxy-2, 3-dimethoxyxanthone(2), 1,3,6-trihydroxy-2, 7-dimethoxyxanthone(3),7-hydroxy-1,2, 3 -trimethoxyxanthone (4), 1,2,3, 6, 7 pentamethoxyxanthone(5), 1,3,7-trihydroxy2,6-dimethanoxyxanthone (6),7-hydroxy-1methoxyxanthone(7) and 1,7-dihydroxy-3,4dimethoxyxanthone(8) [6]. Along with jujube seed, polygala was employed as one of the sedative agents in Chinese medicine and their actions had been associated with transmitters such as GABA, serotonin and dopamine [7]. In fact, in studies of Polygala, two groups of compounds most related to sleep and which exert sedative effects are 3,4-methoxycinnamic acid and phenylpropanoids [8,9]. Effects of Polygala on sleep have been studied in mice that demonstrated decreased locomotor activities. Increase of total sleep time and reduced sleep latency after induction by pentobarbital were also observed [10]. In Japan, this chemical from Polygala had been used as an anti-stress agent [11]. Both mechanisms were proposed as the result of 3,4,5-trimethoxycinnamic acid inside the root of Polygala acting as 1) an GABAa agonist 2) an agent which upregulates glutamic acid decarboxylase and 3) an agent which upregulates the gamma subunit of GABAa receptor [10]. Its anxiolytic and sedative effects were further tested in X maze and open field with mice. Again, its synergetic effect was induced with pentobarbital injection which was found to shorten sleep latency and prolong duration of sleep [12]. The saponins and pyrones in Polygala, though less known, would also be sedative and cause hypnosis [12,13]. In addition, Polygala roots produced little toxic effect below $3.94 \mathrm{~g} /$ $\mathrm{Kg}$ [12] although the saponin inside the Polygala might produce gastrointestinal irritation [14]. Sleep, apart from induction time and total sleeping time, is a behavioral process which needs to be deciphered with physiological devices like the electroencephalogram (EEG). In this work, we reported the study of different components of the EEG in the mice under normal (not induced) sleep, and compared with the EEG patterns generated when sleep was induced by 1) Polygala root and 2) a well-established sedative western drug Alprazolam. This is the first time that Polygala was compared and reviewed with other western agents. The EEG of the mice as that of human was complicated and in order to decipher changes, all of the non-rapid eye movement (NREM) sleep waves would be evaluated, and as possible be quantified at a defined period of sleep and be compared. Waveforms to be quantified include the $\mathrm{k}$ waves, the $\mathrm{v}$ wave and the $\mathrm{w}$ waves (indicating stage 2, 3 of sleep), while delta and theta waves indicating deep beginning sleep (stages 3 and stage 1, respectively) [15-21]. Induction time, sleep time (total), and wakeful time within a period of 3 hours of sleep in the mice would also be assessed. There were very few EEG recordings found on mice in literature and the majority was done on diseased models [22]. One of the first EEG recordings of drug treatment was performed by Meerlo et al. who tested gamma hydroxybutyrate as a vigilant and sleeping agent. The conclusion of that work was that gamma hydroxybutyrate worked only on a high dose and did not seem to produce natural form of sleep [23].

Apart from sleep, Polygala had been associated with other CNS benefits which include enhancing learning and memory in mice [24] and improving cognition in the scopolamine treated rat [25]. Studies further indicated that several species of Polygala including $P$. tenuifolia, $P$. senega and $P$. sabulosa would all be used $[4,12,13]$ in different parts of the world.

In western medicine, the classical medications for sleep can be divided into three categories; the first group is the benzodiazepine receptor agonists that include the frequently used temazepam and trizolam. These drugs act on the benzodiazepine sites of the GABA complex and enhance stage 2 sleep. In general, the action is a result of increased inhibition of GABA by shifting of chloride ion leading to hyperpolarization and stabilization of neuronal membranes. The second group of hypnotics includes Zolpidam, zaleplon and Eszoplclone. Zolpidam can be absorbed quickly and is good for induction of sleep, and it acts through omega I receptors of the benzodiazepine group. Zaleplon is also good for the induction of sleep, but it is not related to either diazepine or barbiturate; instead, it interacts with the GABA diazepine complex. Eszoplclone interacts with another GABA receptor site close to the diazepine receptor [26-28]. Zopiclone and related $\mathrm{z}$ compounds are dominating the markets and are nondiazepine hypnotics for treating insomnia. This group of hypnotics not only increases GABA transmissions but also increases low amplitude waves and reduces rapid eye movement (REM) sleep [29,30]. Another group of sedative which is also used for sleep, sedation of anxiety includes the Alprazolam which is grouped with the benzodiazepines [31,32]. This 
latter group of milder sedatives to induce sleep is effective for acute treatments of insomnia [32]. Other agents like melatonin and related compounds that restore diurnal rhythm are also used as insomnia medication, and reported a reduced latency of getting into sleep and the increased duration of sleep [33].

\section{Materials and Methods}

The animal experiments were approved by the Department of Health, HKSAR government. Twelve-week old male ICR (Institute for Cancer Research, Sutton, UK) mice weighing around $30 \mathrm{~g}$ each were used in this study. Mice were individually housed and kept in a room with temperature controlled at $22-25^{\circ} \mathrm{C}$ and under a 12-hour light/dark cycle. Food and water were available ad libitum.

Electroencephalogram setup: Mice were anchored with EEG electrodes on the head in the following coordinates: frontal: $1.7 \mathrm{~mm}$ lateral to midline, $1.5 \mathrm{~mm}$ anterior to bregma; parietal: $1.7 \mathrm{~mm}$ lateral to midline, $1.0 \mathrm{~mm}$ anterior to lambda. The electrodes were mounted to the skull by dental cement and later connected to a connector plug during recording. The mice were connected to the EEG setup for another 8 days of habituation and received the following treatment for 8 days.

Extraction of herbal formula: Roots of Polygala tenuifolia were bought from local pharmacy in Hong Kong and crushed into powder with grinding machine, followed by boiling in deionized water $(5 \mathrm{~g}$ to $120 \mathrm{ml}$ ) for 60 minutes. Decoction was filtered and freeze dried for storage and dissolved in $0.9 \%$ saline upon usage [38].

Mice were divided into 4 groups and treatments were given to mice orally every day for 8 days. The treatments were $0.9 \%$ Saline $(5 \mathrm{ml} / \mathrm{kg} /$ day $)$, Alprazolam $(0.6 \mathrm{mg} / 5 \mathrm{ml} / \mathrm{kg} /$ day $)$, Polygala herbal formula $(0.374 \mathrm{~g} / 5 \mathrm{ml} / \mathrm{kg} /$ day $)$, and Alprazolam $(0.6 \mathrm{mg} / 5 \mathrm{ml} / \mathrm{kg} /$ day $)$ plus herbal medicine $(0.374 \mathrm{~g} / 5 \mathrm{ml} / \mathrm{kg} /$ day $)$. All drugs were dissolved in $0.9 \%$ saline and given in 5 $\mathrm{ml} / \mathrm{kg} /$ day. After the 8th day's treatment, EEG signals were recorded by a signal amplifier and data requisition system (Tongren Technologies, China) for 3 hours. For each of the three groups of animals ( $n=3$ to 4 animals for each group), namely saline control, polygala herbal formula and Alprazolam, EEG was measured in every animal every 15 minutes, each for 30 seconds in the first hour of sleep and the same for the second and third hours. For comparison of wave forms/ durations, only the 30 second strips of the last 45 minutes of each hour was used. Three recording strips were selected from each of the three animals from each group for analysis. The recording experiments were repeated two times.

\section{Results}

Analyzing the complex waveforms is difficult and the investigators relied on the defined waveforms in the literatures as specified before in this paper. Only the non-REM (NREM) sleep was analyzed. The sleep cycles of the mice in our experiments usually lasted for around three hours and hence the EEG was recorded for 120 seconds when they were in the first hour, second hour and the third hour of sleep. Electrodes were anchored previously onto the skull at two defined positions on the days before recording so that they got habituated to the devices. During sleep the lengths of $\mathrm{k}$ complexes, delta and theta waves were recorded. Theta waves (stages 1 to 2 sleep) and delta and $\mathrm{k}$ waves (stage 3 deep sleep) (Figure 1a,b) represent total sleep waves while those of $\mathrm{v}$ and $\mathrm{w}$ waves (Figure 1c), as well as saw-edged waves (drug related beta wave) were defined as stage 2 (superficial or beginning of deep sleep) [34] (Figure 1d). The rest of the sleep periods would compose of sleep spindles (Figure 1e) or other mixed waves. We had focused on the theta, $\mathrm{v}$ wave, $\mathrm{k}$ wave, and delta wave that were the most important components of the sleep cycle.

Our cohort of mice usually slept in the morning after food intake. The sleeping duration was usually for more than 3 hours. The behavioral pattern of actual sleep recorded was that the eyes were closed and the tail was curved and hanging relaxed at the side of the body with the tip of the tail pointing forward. At the time, theta waves began to appear in the EEG as well. If they were put into the dark chamber for sleep at 10:30 in the morning, the usual induction time to total motionless deep sleep as recorded was $50 \mathrm{~min}$ \pm 3 minutes $(n=3)$ whilst induction time after feeding Alprazolam was $31 \pm 7.5$ minutes and feeding the herbal formula with Polygala was $25 \pm 0$ minutes. The feeding time of the latter two medications was also set at 10:30 am. After feeding of the medication, the mice too were returned to the dark chambers for video recording on sleep. At the same time, EEG 


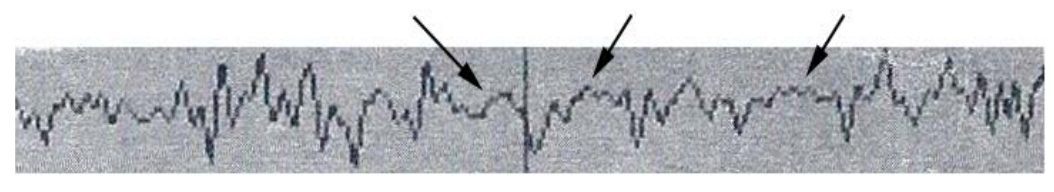

Figure 1a: Theta wave (arrows).

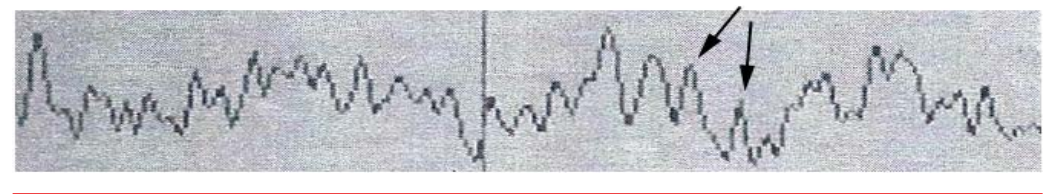

Figure 1b: Delta waves (arrows).

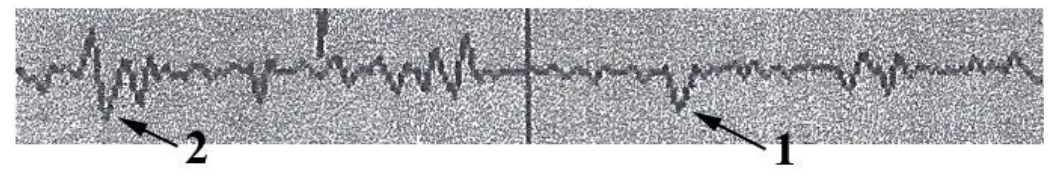

Figure 1c: 1 indicates $V$ wave. 2 indicates $K$ wave.

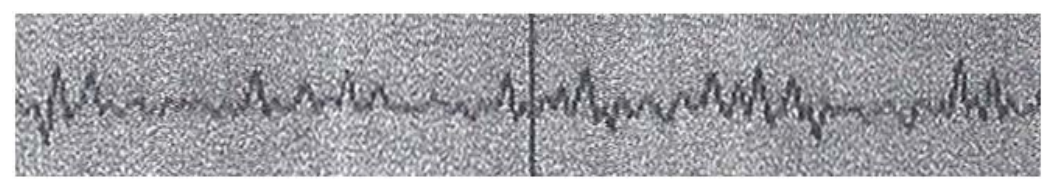

Figure 1d: Saw-edge beta wave.

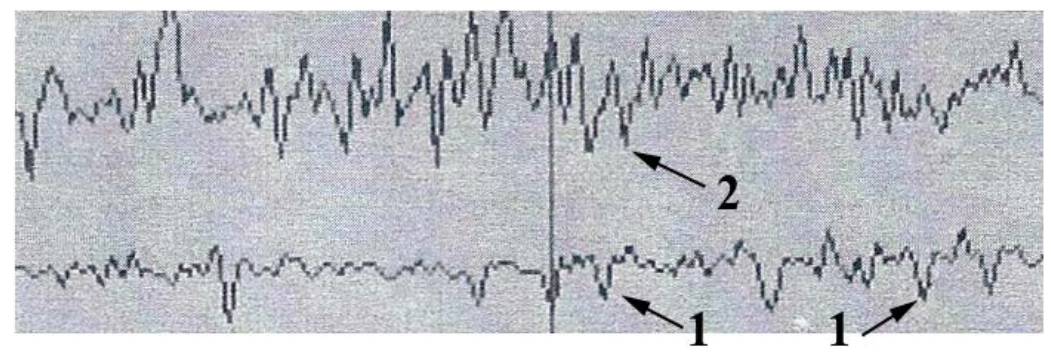

Figure 1e: 1 indicates $V$ waves. 2 indicates sleep spindle.

recording was performed as in the materials and methods.

Using the EEG wave, the numbers of $\mathrm{v}$ and $\mathrm{w}$ waves were counted within strips of 30 seconds once every hour in all groups of mice which represented the stage 2 sleep (beginning of deep sleep). These results from the Alprazolam group, the herbal group, the control group presented were respectively $20.5 \pm 3.8$ per 30 seconds in the Alprazolam group, $20.4 \pm 3.16$ per 30 seconds in the Polygala group and $14.6 \pm 3.3$ per 30 seconds in the control $(n=9)$. These results indicated that both Polygala and Alprazolam were better than control in initiating v/w waves sleep (beginning of deep sleep). However, preliminary segmental studies appeared to indicate the Polygala and Alprazolam had different advantage over the control: the Alprazolam acted on the initial first hour better $(20 \mathrm{v} / \mathrm{w}$ waves in Alprazolam versus $16 \mathrm{v} / \mathrm{w}$ waves in control in 30 second strip in the first while the Polygala appeared to do better than control in the subsequent hours (e.g. average $23 \mathrm{v} / \mathrm{w}$ waves in Polygala versus 17 $\mathrm{v} / \mathrm{w}$ waves in control in 30 seconds during the third hour of sleep). Likewise, when Alprazolam was combined with the herb in the treatment of sleep, the sleep markers increased compared with just using Alprazolam alone ( $40 \pm 8.0 \mathrm{v} / \mathrm{w}$ waves for Alprazolam plus polygala versus $21 \pm 2.2 \mathrm{v} / \mathrm{w}$ waves for Alprazolam per 30 second strip).

When one compared the theta wave (designated as a wave form for first stage of sleep (i.e. light sleep) $[35,37]$ of the EEGs of mice fed with Polygala, Alprazolam and control saline and the recordings were taken once every hour (starting from the 45th minute of the first hour) for three hours, theta wave duration was found to increase in the first hour, decreased substantially in the second hour and increased again in the third hour (Figure 2). When the duration of theta waves per unit time was compared between the three groups of Polygala, Alprazolam and control saline, the theta durations in Polygala fed and Alprazolam fed animals per 30 seconds of recording in the first and second hours of sleep were higher than that of control (Figure 2). By the third hour, the theta duration per $30 \mathrm{sec}$ was still higher in the EEG of Polygala fed mice than that of control (Figure 2).

When the theta duration representing light sleep and the delta duration representing deep sleep of stage 3 (now denoted as N3 stage since stage 4 had been taken out [36]) were added together, in the saline control as per 30 seconds, the total theta plus delta duration of the first hour was $9.67 \pm 0.58 \mathrm{sec}$, the second hour was 7.83 \pm 0.29 seconds in the second hour and $8.33 \pm$ $0.58 \mathrm{sec}$ in the third hour. For the Polygala fed mice, theta plus delta duration was $16.17 \pm 0.29$ seconds in the first hour, $12.17 \pm 0.58$ seconds in the second and $11.67 \pm 0.58$ in the third hour. In the Alprazolam group, the theta plus delta duration was $11.67 \pm 1.04$ seconds in the first hour, $7.5 \pm 0.50$ seconds in the second hour and $7.00 \pm 0$ seconds in the third hour. There was an obvious increase in the duration of theta plus delta waves in the Polygala and Alprazolam treated groups, while the theta plus delta duration 
was slightly longer in the Polygala group than the Alprazolam group. When the Alprazolam treated group was added with Polygala, the theta plus delta wave duration increased to $13.17 \pm$ 1.26 seconds in the first hour, $10 \pm 2$ seconds in the second hour and $8.67 \pm 0.58$ seconds in the third. It was better than treatment of Alprazolam alone but did not reflect a simple addition. The comparisons were illustrated in Figure 3.

When the number K complex [37] in the EEGs were counted per 30 seconds in the three groups, bearing in mind that $\mathrm{K}$ complex occurred in stage 3 deep sleep, again the Polygala fed mice reported $9.00 \pm 1.00 \mathrm{~K}$ wave at the first hour, $8.00 \pm 1.73$ in the second hour and $6.33 \pm 1.53$ in the third hour while in the Alprazolam group, the $\mathrm{K}$ complex number was $7.33 \pm 1.53$ in the first hour and $6.34 \pm 0.59$ in the second hour and $4.8 \pm$ 1.1 in the third hour. Those fed with control saline was $4.00 \pm 1.73$ in the first hour, $3.33 \pm 1.15$ in the second hour and $3.00 \pm 1.00$ in the third hour. There was an obvious upregulation of Polygala and Alprazolam treated animals. The comparisons were illustrated in Figure 4.

\section{Discussion}

Our results indicated that both the herb of Polygala and Alprazolam could benefit sleep via the shortening of sleep induction time and the increase in wave forms of EEG related to both stages 1, 2 and stages 3 of the NREM sleep. The increase of theta waves, $v$ waves, delta waves as well as $\mathrm{k}$ complex in either duration or number proposed that deeper or better quality sleep occurred in the experimental animals on both herbal and western hypnotics. As well the additional of the Chinese herb together with Alprazolam would produce an enhanced effect over the use of a single agent, noting however, that the combined effect is not a simple addition of the two agents. This is the first time that a Chinese herbal agent has been compared with a well-established sedative of the benzodiazeprne group and also the first time when comparison was performed with various wave forms of the EEG. This study illustrated that herbal agents could have at least similar efficacy in the induction of sleep as the manufactured western drugs. In some aspects (e.g. total theta and delta durations) Polygala might look perhaps better than Alprazolam. Although in this study that the herbal agent appeared in some criteria to have slightly better effect than Alprazolam on these aspects of sleep, one would have to be

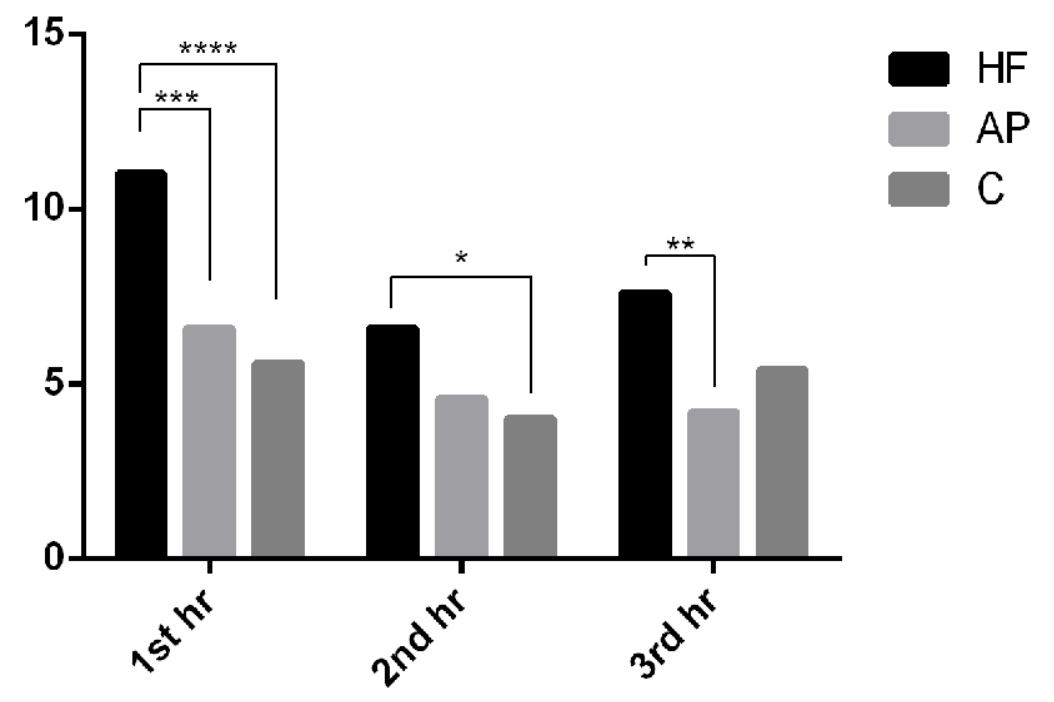

Figure 2: Shows duration (seconds) of theta waves appeared per 30 second strip during the first three hours of sleep under different treatments. HF represents Polygala herbal formula; AP represents Alprazolam; $C$ represents controls. Means with standard deviation. Statistical analyses were performed using two-way repeated measures ANOVA, $F(2,12)=32.30, p<.0001$, followed by Tukey's test.

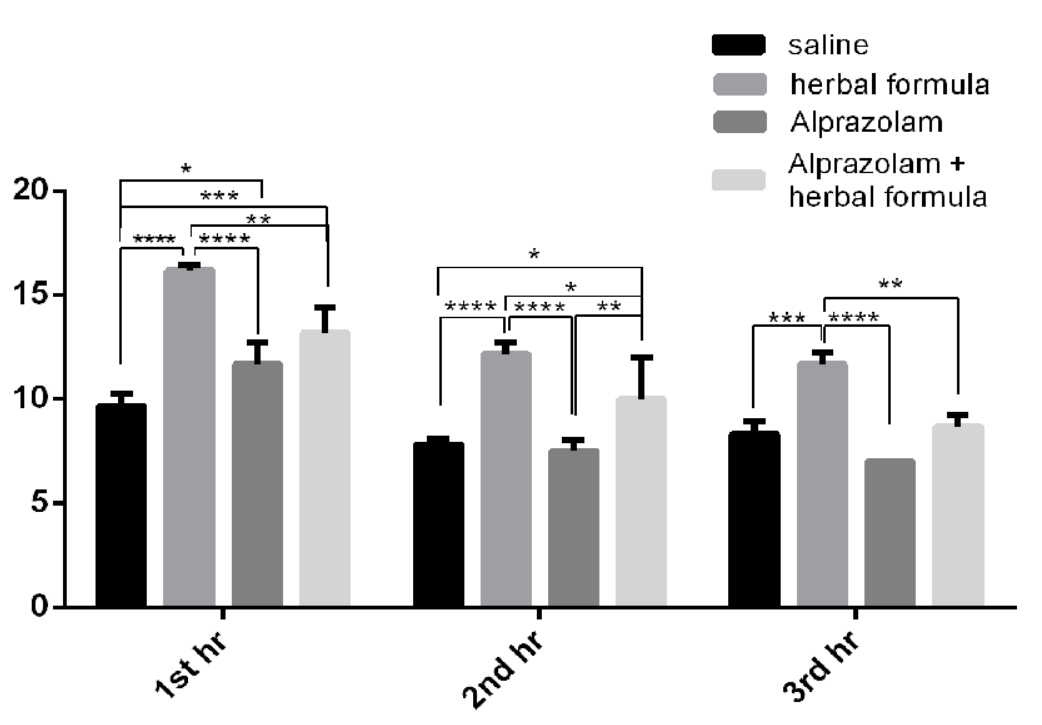

Figure 3: Shows duration (seconds) of delta plus theta waves appeared per 30 second strip during the first three hours of sleep under different treatments. Means with standard deviation. Statistical analyses were performed using two-way repeated measures $\operatorname{ANOVA}, F(3,8)=24.75, p=$ .0002 , followed by Tukey's test.

careful in comparison because there are many group of anti-insomnia drugs on the market and Alprazolam is one of the drugs that would have a sedative effect and works well initially in naïve patients (not treated before) for insomnia [28]. This study also indicated the possible values of the individual markers (waves) in the EEG that can be of value in sleep experiments.

Preliminary studies in this laboratory when trying to counteract the insomniac ability of caffeine by 


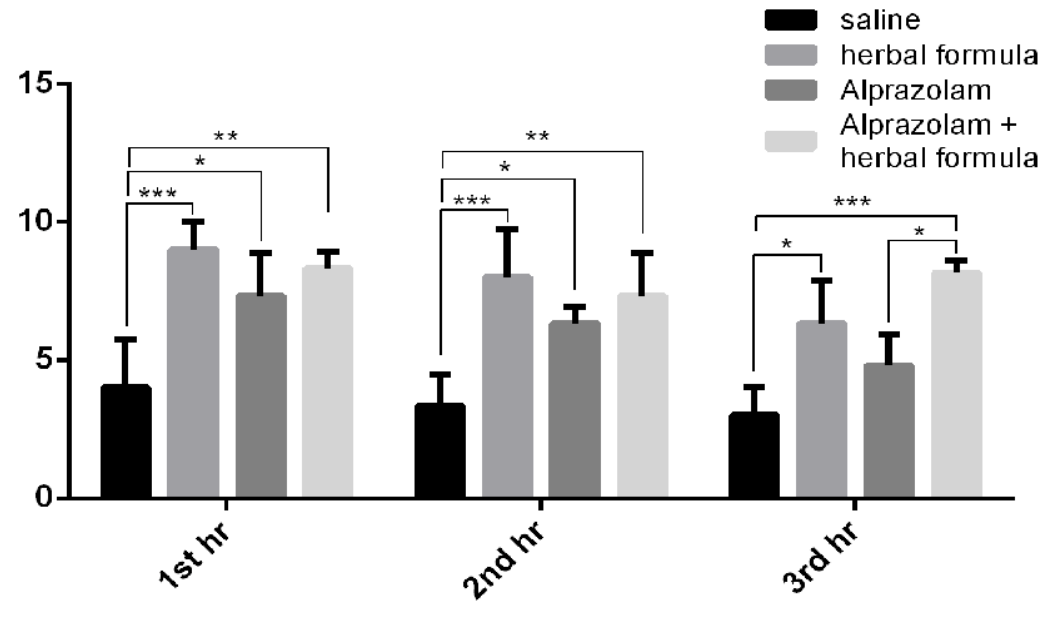

Figure 4: Shows number of $\mathrm{k}$ complex appeared in a 30 second strip during the first, three hours of sleep under different treatments. Means with standard deviation. Statistical analyses were performed using two-way repeated measures ANOVA, $F(3,8)=9.430, p=$ .0053 , followed by Tukey's test. adding either our herb or melatonin to caffeine treated animals ended up with disappointing results. Although the sleep markers in the EEG after consumption of our herb or melatonin appeared to upregulate sleep markers subsequent to caffeine consumption, the induction time was terribly long (hours) which did not justify the usage of these latter agents for reversal of the insomniac effect of caffeine (data not shown). This is the first evidencebased comparison on the effects of herbal agents with western sedatives/hypnotics on the potential benefit for insomnia. Though not exhaustive, this may be one of the directions where herbal medication can clarify their scientific values, via comparison of western therapeutic agents. Hopefully this is a possible way of mordernizing herbal medicine amongst others to take a stride forwards.

\section{References}

1. Hong $F$, Wang $L$, Wu SL, et al. A review of three commonly used herbs which enhance memory and new evidences which show their combination could improve memory in young animals. Mini. Rev. Med. Chem 17(16), 1537-1547 (2016).

2. Wang CM, Liang W, Yew DT. Comparison of two old phytochemicals versus two newly researched plant-derived compounds: Potential for brain and other relevant ailments. Evid. Based. Complement. Alternat. Med 682717 (2014).

3. Zhang L, Lam WP, Lü L, et al. How would composite traditional Chinese medicine protect the brain--an example of the composite formula "Pien Tze Huang". Curr. Med. Chem 18(1), 3590-3594 (2011)

4. Sharma G, Jhade A, Malviya S, et al. Ethnopharmacological attributes of Polygala senega. IJPLS 6(1), 4202-4208 (2015).

5. Zhang YH, Bai L, Li Z, et al. HPLC fingerprint and active components determination of Polygala tenuifolia root bark and root. Zhong. Yao. Cai 38(1), 1408-1412 (2015).

6. Xu LL, Li CJ, Yang JZ, et al. Chemical constituents of Polygala tenuifolia root. Zhong. Yao. Cai 37(1), 1594-1596 (2014).

7. Wang Q, Wang LW, Liu XM. Brief review about compatibility and their pharmacological effects of Chinese material medica as tranquilizer. Zhongguo. Zhong. Yao. Za. Zhi 32(1), 2342-2346 (2007).

8. Nugroho A, Kim MH, Choi J, et al. Phytochemical studies of the phenolic substances in Aster glehni extract and its sedative and anticonvulsant activity. Arch.
Pharm. Res 35(1), 423-430 (2012).

9. Tu Y, Cheng SX, Sun HT, et al. Ferulic acid potentiates pentobarbital-induced sleep via the serotonergic system. Neurosci. Lett 525(1), 95-99 (2012).

10. Lee $\mathrm{Cl}$, Han JY, Hong JT, et al. 3,4,5-Trimethoxycinnamic acid (TMCA), one of the constituents of Polygalae radix enhances pentobarbital-induced sleeping behaviors via GABAAergic systems in mice. Arch. Pharm. Res 36(1), 1244-1251 2013.

11. Kawashima K, Miyako D, Ishino Y, et al. Antistress effects of 3,4,5-trimethoxycinnamic acid, an active constituent of roots of Polygala tenuifolia (Onji). Biol. Pharm. Bull 27(1), 1317-1319 (2004).

12. Yao Y, Jia M, Wu J, et al. Anxiolytic and sedative-hypnotic activities of polygalasaponins from Polygala tenuifolia in mice. Pharm. Biol 48(1), 801-807 (2010).

13. Duarte FS, Duzzioni M, Mendes BG, et al. Participation of dihydrostyryl-2-pyrones and styryl-2-pyrones in the central effects of Polygala sabulosa (Polygalaceae), a folk medicine topical anesthetic. Pharmacol. Biochem. Behav 86(1), 150-161 (2007).

14. Wen $L$, Xia, N, Tang $P$, et al. The gastrointestinal irritation of polygala saponins and its potential mechanism in vitro and in vivo. Biomed. Res. Int 918048 (2015).

15. Loomis AL, Harvey EN, Hobart GA. Cerebral states during sleep, as studied by human brain potentials. J. Exp. Psychol 21(1), 127 (1937).

16. Green JD, Arduini AA. Hippocampal electrical activity in arousal. J. Neurophysiol 17(1), 533-557 (1954).
17. Vanderwolf $\mathrm{CH}$. Hippocampal electrical activity and voluntary movement in the rat. Electroencephalogr. Clin. Neurophysiol 26(1), 407-418 (1969).

18. McCormick L, Nielsen T, Nicolas A, et al. Topographical distribution of spindles and K-complexes in normal subjects. Sleep 20(1), 939-941 (1997).

19. Halasz P. K-complex, a reactive EEG graphoelement of NREM sleep: an old chap in a new garment. Sleep Med. Rev 9(1), 391412 (2005).

20. Pilon M, Zadra A, Joncas S, et al. Hypersynchronous delta waves and somnambulism: brain topography and effect of sleep deprivation. Sleep 29(1), 7784 (2006).

21. Cash SS, Halgren E, Dehghani N, et al. The human K-complex represents an isolated cortical down-state. Science 324(1), 10841087 (2009).

22. Vyazovskiy VV, Deboer $\mathrm{T}$, Rudy B, et al. Sleep EEG in mice that are deficient in the potassium channel subunit K.v.3.2. Brain. Res 947(1), 204-211 (2002).

23. Meerlo $P$, Westerveld $P$, Turek FW, et al. Effects of gamma-hydroxybutyrate (GHB) on vigilance states and EEG in mice. Sleep 27(1), 899-904 (2004).

24. Xue W, Hu F, Yuan, et al. Polygalasaponin XXXII from Polygala tenuifolia root improves hippocampal-dependent learning and memory. Acta. Pharmacol. Sin 30(1), 12111219 (2009).

25. Sun XL, Ito H, Mazurka T, et al. Effect of Polygala tenuifolia root extract on scopolamine-induced impairment of rat spatial cognition in an eight-arm radial 
Sleep Induced by a Herbal Agent Polygala tenuifolia versus that by Alprazolam: A Review with New Data Research on EEG and Sleep Duration

maze task. Biol. Pharm. Bull 30(1), 1727-1731 (2007).

26. Gillin JC, Byerley WF. Drug therapy: The diagnosis and management of insomnia. $N$. Engl. J. Med., 322(1), 239-248 (1990).

27. Elie R, Rüther E, Farr I. Sleep latency is shortened during 4 weeks of treatment with zaleplon, a novel non-benzodiazepine hypnotic. Zaleplon Clinical Study Group. J. Clin. Psychiatry 60(1), 536-544 (1999).

28. Schuen JN, Millard SL. Evaluation and treatment of sleep disorders in adolescents. Adolesc. Med 11(1), 605-616 (2000).

29. Trachsel L, Dijk DJ, Brunner DP. Effect of zopiclone and midazolam on sleep and EEG spectra in a phase-advanced sleep schedule. Neuropsychopharmacology 3(1), 11-18 (1990).
30. Mann K, Bauer H, Hiemke C. Acute, subchronic and discontinuation effects of zopiclone on sleep EEG and nocturnal melatonin secretion. Eur. Neuropsychopharmacol 6(1), 163-168 (1996).

31. Straw RN. Brief review of published alprazolam clinical studies. Br. J. Clin. Pharmacol 19(Suppl 1), 57S-59S (1985).

32. Kales A, Bixler EO, Vela-Bueno A. Alprazolam: effects on sleep and withdrawal phenomena. J. Clin. Pharmacol 27(1), 508-515 1987.

33. Brzezinski A, Vangel MG, Wurtman RJ, et al. Effects of exogenous melatonin on sleep: A meta-analysis. Sleep. Med. Rev 9(1), 41-50 (2005).

34. Cicogna $P$, Natale V, Occhionero $M$, et al. Slow wave and REM sleep mentation. Sleep. Res.
Online 3(1), 67-72 (2000).

35. Pinel J. Biopsychology. InAllyn and Bacon: Needham Heights MA, (1992).

36. Steriade M. Grouping of brain rhythms in corticothalamic systems. Neuroscience 137(1), 1087-1106 (2006)

37. Pedley TA. Electroencephalography. Basic principles, clinical applications and related fields, 3rd edition: E. Niedermeyer and F. Lopes da Silva (Eds.) (Williams and Wilkins, Baltimore, MD, (1993).

38. Qiu Y, Wang LG, Jia YF, et al. Effects of the crude extract of Polygala tenuifolia willd on human sperm in vitro. Journal. Of. Zhejiang. University. Science. B 12(6), 448-454 (2011). 